\section{Seroprevalencia de Bartonella henselae en personas con riesgo ocupacional}

\author{
Ignacio Troncoso, Christof Fischer, Francisca Arteaga, \\ Cristian Espinoza, Teresa Azócar y Katia Abarca
}

\section{Seroprevalence of Bartonella henselae in occupational risk persons}

Introduction: Bartonella henselae infection is a worldwide zoonosis with the domestic cat as reservoir. Although people with occupational contact with these pets are risk population only few studies of prevalence in them have been reported. Methods: A study of seroprevalence of $B$. henselae was performed to veterinaries and other persons with occupational contact with cats, residents from the Bío-Bío region of Chile. Serum IgG antibodies against $B$. henselae were determined by indirect immunofluorescence (IFI). Demographic data and history of cat bites or scratches were recorded. Results: There were 76 persons included in the study, 18 to 69 years old. A $93.4 \%$ had a history of cat scratch or bite. A seroprevalence of $60.5 \%$ were found. No differences were found between gender, age, or history of cat scratch or bite. Conclusions: A high seroprevalence in people from this region with occupational risk were found. No subgroups with higher risk factors than others were identified.

Key words: Seroprevalence, Bartonella henselae, occupational risk, immunofluorescence.

Palabras clave: Seroprevalencia, Bartonella henselae, riesgo ocupacional, inmunofluorescencia.

\section{Introducción}

$\mathrm{H}$ istóricamente los animales de compañía han desempeñado un rol importante en la vida del hombre; varios estudios demuestran los beneficios para la salud física y psicológica. A pesar de estos beneficios existen perjuicios, tales como el riesgo de mordeduras, alergias $\mathrm{y}$ transmisión de enfermedades conocidas como zoonosis ${ }^{1}$.

Una de estas enfermedades, de amplia distribución mundial, es la infección producida por Bartonella henselae, una bacteria gramnegativa, multiflagelar, pleomórfica, aeróbica e intracelular facultativa ${ }^{2}$. Su reservorio en la naturaleza es el gato doméstico y su transmisión al hombre ocurre a través de la mordedura o arañazo. Entre los félidos la infección se transmite por las pulgas. Se ha planteado la posibilidad de que también la transmitirían al hombre, lo que no ha sido demostrado ${ }^{3}$.

La enfermedad es de curso autolimitado, relativamente benigna, puede afectar a personas de cualquier edad, presentando una predilección por

\footnotetext{
Universidad Santo Tomás, Sede Concepción, Chile. Escuela Medicina Veterinaria (IT, CF, FA, CE). Pontificia Universidad Católica de Chile, Santiago, Chile. Laboratorio de Virología e Infectología Molecular (KA, TA).

Los autores declaran no tener conflictos de interés.

Fuente de financiamiento: Escuela de Medicina Veterinaria de la Universidad Santo Tomás, sede Concepción.

Recibido: 4 de junio de 2015 / Aceptado: 26 de enero de 2016

Correspondencia a:

Ignacio Troncoso T.

ignaciotroncoso@santotomas.cl
}

niños y adolescentes. Las manifestaciones clínicas incluyen la enfermedad por arañazo de gato (EAG) típica caracterizada por una linfoadenopatía regional subaguda, y la formas atípicas, que puede afectar prácticamente a cualquier órgano: corazón, hígado, bazo, hueso, médula ósea, sistema linfático, músculo, tejidos blandos y sistema nervioso central ${ }^{4}$. En el caso del paciente inmunocomprometido, principalmente aquellos con inmunodeficiencia celular, la respuesta a la infección es vasoproliferativa y de mayor diseminación hemátogena incluyendo la angiomatosis bacilar ${ }^{5}$ peliosis hepática ${ }^{6}$ endocarditis bacteriana y la bacteriemia persistente. En cambio, en el paciente inmunocompetente, la respuesta a la infección es de tipo granulomatosa supurativa crónica ${ }^{7}$.

El diagnóstico de esta enfermedad se ha sustentado principalmente en manifestaciones clínicas, epidemiológicas, de laboratorio y ocasionalmente histológicas. Se ha sugerido que la presencia de al menos tres de los siguientes criterios sustentan el diagnóstico: linfoadenopatía regional con la exclusión de otras etiologías habituales; antecedente de contacto con gato con o sin lesión de inoculación primaria; prueba serológica positiva para anticuerpos contra $B$. henselae, siendo la técnica más sensible la inmunofluorescencia indirecta (IFI); histopatología característica consistente en una linfoadenitis granulomatosa crónica supurativa con la presencia de bacilos teñidos con la tinción argéntica de Warthin-Starry y la prueba de reacción de polimerasa en cadena (RPC) positiva para B. henselae . $^{8}$

Respecto a la epidemiología, estudios realizados en niños han mostrado prevalencias de $15 \%$ en Grecia y $18,5 \%$ en Canadá; en adultos las cifras varían entre 3 y 7\% en E.U.A., Hawaii, España, Tailandia y Japón, y 37\% en Canadá ${ }^{3}$. Estudios efectuados en médicos veterinarios en Austria y Japón y en propietarios de gatos en Alemania muestran prevalencias de 51, 15 y $79 \%$; respectivamente ${ }^{9-11}$.

Hasta la fecha, en nuestro medio se han publicado varios casos o series clínicas de infecciones por $B$. henselae ${ }^{12-17}$, incluyendo un estudio en propietarios de felinos ${ }^{18}$, otro en niños y en una población de riesgo ocupacional $^{19}$. En estos últimos, se ha reportado una prevalencia de $18 \%$ en propietarios de gatos; $13,3 \%$ en niños y de $10,3 \%$ en adultos con riesgo ocupacional de las ciudades de Santiago y Valdivia ${ }^{18,19}$. Debido a la limitada información existente en el país, se realizó este estudio para determinar la seroprevalencia por $B$. henselae en un grupo de riesgo ocupacional de una región de la zona centro sur del país.

\section{Método}

Se invitó a participar a dos grupos de personas con riesgo ocupacional: médicos veterinarios pertenecientes a organizaciones gremiales de las ciudades de Concepción y Chillán; y criadores de gatos, cuya ocupación incluía un alto contacto con felinos. El cálculo del tamaño de muestra se realizó en el programa Epi 6, utilizando la fórmula de poblaciones infinitas, con una proporción esperada de $10,3 \%$ según el último antecedente nacional ${ }^{19}$, con un nivel de confianza de $95 \%$ y una precisión de $7 \%$. Se obtuvo como resultado un número de 72 muestras. Luego de firmar un consentimiento informado se realizó la toma de muestra de sangre para la obtención de sueros, los que fueron almacenados a $-20{ }^{\circ} \mathrm{C}$ hasta su procesamiento.

A cada sujeto se aplicó una encuesta que recopiló información de datos epidemiológicos, tales como: género, edad, antecedentes de accidentes con gatos (arañazo o mordedura), y signos clínicos compatibles con la EAG (fiebre, lesión de inoculación, linfadenopatía regional) o signos de infección local. 
Se determinó la presencia de anticuerpos séricos IgG específicos contra $B$. henselae por la técnica de IFI, utilizando un kit comercial (Focus ${ }^{\mathrm{TM}}($ ). Las muestras fueron procesadas en el Laboratorio de Infectología y Virología Molecular de la Pontificia Universidad Católica de Chile siguiendo las instrucciones del fabricante. En cada procedimiento se utilizó un control negativo y uno positivo.

El título de corte recomendado por los Centers for Disease Control and Prevention de E.U.A (CDC) para el diagnóstico de infección es de 1/64; sin embargo, en este laboratorio nacional, debido a la elevada seropositividad encontrada en niños y personas con riesgo ocupacional en nuestro medio ${ }^{19}$, se estableció un título de corte en dilución de $1 / 256$. Si bien para estudios de seroprevalencia el punto de corte más utilizado es $1 / 64$, considerando la elevada seroprevalencia en veterinarios ${ }^{9-11}$, en este estudio se utilizó una dilución de 1/256.

Para el análisis de los datos se calcularon las frecuencias porcentuales y para la comparación de la prevalencia según género, edad, antecedentes de mordedura y/o rasguño, y signología clínica se utilizaron test no paramétricos (Chi cuadrado o Fischer).

\section{Resultados}

Se ingresaron al estudio 76 personas con riesgo ocupacional de la región: 40 veterinarios y 36 criadores de felinos; 37 hombres y 39 mujeres. El rango de edad en los hombres fue de 18 a 53 años (mediana: 36), y de 19 a 69 años (mediana: 33) para las mujeres.

En 46 sueros se la identificó la presencia de anticuerpos IgG anti- $B$. henselae, lo cual equivale a una seroprevalencia de $60,5 \%$. No se observó diferencia según género: las mujeres presentaron una prevalencia de 58,3\% (21/39) y los hombres de 67,5\% (25/37); ni según el grupo etario: los menores de 35 años tuvieron una prevalencia de 70\% (28/40) y los mayores de 35 años de $50 \%(18 / 36)$.

Un total de 71 personas $(93,4 \%)$ manifestaron haber experimentado mordeduras o rasguños de gato alguna vez en su vida y $45(52,5 \%)$, haber presentado signos sugerentes de EAG (lesión de inoculación y/o adenopatía regional) o signos de infección de la herida.

No se encontraron diferencias estadísticamente significativas entre veterinarios y criadores de felinos, entre personas con y sin antecedentes de arañazo o mordedura por gatos, ni entre personas con antecedentes de lesiones sugerentes de EAG o de infección local (Tabla 1).

Tabla 1. Seroprevalencia de Bartonella henselae según factores de riesgo, en 76 sujetos con riesgo ocupacional

\begin{tabular}{lccc}
\hline Características de los sujetos & Positivo/total & $\%$ & $\mathbf{p}^{* *}$ \\
Veterinarios & $20 / 40$ & 50 & 0,06 \\
Criadores felinos & $26 / 36$ & 72,2 & \\
$\begin{array}{l}\text { Antecedentes de accidentes con gatos } \\
\text { (arañazo, mordedura) }\end{array}$ & $43 / 71$ & 60,5 & 0,66 \\
$\begin{array}{l}\text { Signología clínica compatible con EAG o } \\
\text { infección local }\end{array}$ & $28 / 45$ & 62,2 & 0,81 \\
\hline *EAG: Enfermedad por arañazo de gato. **Test de Fisher de dos colas. & \\
\hline
\end{tabular}

\section{Discusión}

La prevalencia de los anticuerpos contra $B$. henselae en este grupo de sujetos con riesgo ocupacional fue bastante superior a lo comunicado en un estudio previo realizado en el país en una población similar (10,3\% de seropositividad en 107 personas que trabajaban en clínicas veterinarias y bioterios de la Región Metropolitana y Valdivia) ${ }^{19}$, así como lo reportado en Ohio (7\% de positividad en 351 médicos veterinarios, técnicos veterinarios y trabajadores de un hospital veterinario $)^{21}$; pero similar a lo reportado en un estudio realizado en Austria (51\% en 137 médicos veterinarios) $)^{9}$ y otro en Alemania ( $79 \%$ de 59 miembros de protectoras de gatos) ${ }^{11}$.

La significativa mayor prevalencia encontrada en este estudio en relación al estudio nacional previo ${ }^{19}$ ocurrió a pesar de haber usado dos diluciones mayores a los anteriores estudios. De haberse usado una dilución de 1/64 se habría obtenido una seroprevalencia aún mayor. Posibles explicaciones a esta mayor prevalencia no parecen estar en la frecuencia de rasguño o mordedura de gato, puesto que en ambos estudios este antecedente fue muy similar, pero sí podrían radicar en las medidas preventivas implementadas después del accidente (por ejemplo, lavado inmediato de las heridas). Si bien esto no fue evaluado en forma directa en este estudio, la mayor frecuencia de signos inflamatorios locales o infección podría ser una evidencia indirecta de menores medidas de precaución o lesiones de mayor intensidad. Efectivamente, en nuestra serie $52,5 \%$ presentó algún tipo de inflamación local o signos de infección en la herida post-rasguño o mordedura, contrastando con $19,6 \%$ en el estudio de Ferrés y cols ${ }^{19}$.

En relación al género, dos estudios han encontrado una mayor seroprevalencia en mujeres ${ }^{10,19}$, mientras que, otro la encuentra mayor en el género masculino ${ }^{21}$, similar a lo obtenido en esta investigación.

Respecto a la edad, en la población general está bien documentado que la EAG se presenta con mayor frecuencia en niños ${ }^{23-25}$. En los adultos de riesgo existe escasa información respecto a la distribución etaria. Tanto el presente estudio como uno realizado en Tailandia ${ }^{22}$ han mostrado una tendencia a una mayor prevalencia en individuos menores a 35 años, sin ser estadísticamente significativa.

Es interesante mencionar que en nuestro estudio cinco personas afirmaron nunca haber sufrido ningún tipo de lesión por rasguño o mordida, sin embargo, tres de ellos tenían evidencia serológica de contacto con el agente. Posibles explicaciones a ello serían haber sufrido una lesión en algún momento lejano el cual no recuerden, o que hayan tenido otras fuentes de infección, como los perros, que han sido documentados como fuente de infección a humanos ${ }^{26,27}$ o mediante vectores como la pulga de gato (Ctenocephalides felis) ${ }^{28,29}$, que se ha propuesto como un posible vector para las personas.

En conclusión, se logró evidenciar una elevada seroprevalencia para $B$. henselae en personas con riesgo ocupacional de la Región del Bío Bío, que mantienen un contacto estrecho con gatos, sin identificarse condiciones o factores que determinen un mayor riesgo dentro de esta población.

Agradecimientos. A todos los profesionales y personas que participaron de forma voluntaria en este trabajo, y al personal del Laboratorio de Infectología y Virología Molecular de la Pontificia Universidad Católica de Chile por el apoyo en el estudio serológico.

\section{Resumen}

Introducción: La infección por Bartonella henselae es una zoonosis de distribución mundial cuyo reservorio es el gato doméstico. Las personas en 
contacto con estas mascotas constituyen una población de riesgo, existiendo escasos estudios de prevalencia en ellos. Método: Se realizó un estudio de seroprevalencia de $B$. henselae en un grupo de médicos veterinarios e individuos con contacto ocupacional con gatos, residentes de la Región del Bío-Bío. Se determinó la presencia de anticuerpos séricos tipo IgG específicos mediante la técnica de inmunofluorescencia indirecta (IFI), en sueros diluidos hasta $1 / 256$. Se realizó una encuesta que registró datos demográficos y antecedentes de mordeduras o rasguños. Resultados: Se estudiaron 76 personas, entre 18 y 69 años de edad. El 93,4\% de los individuos tenía antecedentes de rasguño o mordedura por gatos. Se encontró una seroprevalencia de $60,5 \%$. La seropositividad no fue diferente según género, edad, ni en aquellos con o sin antecedente de mordedura o lesiones sugerentes de enfermedad por arañazo de gato. Conclusiones: Se demostró una elevada seroprevalencia en personas con riesgo ocupacional en esta región. No se identificaron subgrupos con mayores factores de riesgo que otros.

\section{Referencias bibliográficas}

1.- Dabanch J. Zoonosis. Rev Chilena Infectol 2003; 20 (Supl 1): S47-S51.

2.- Chomel B B, Boulois H J, Muruyama S, Breitschwerdt E. B. Bartonella spp. in pets and effect on human health. Emerg Infect Dis 2006; 12: 389-94.

3.- Ferrés M, Abarca K, Godoy P, García P, Palavecino E, Méndez G, et al. Presencia de Bartonella henselae en gatos: cuantificación del reservorio natural y riesgo de exposición humana de esta zoonosis en Chile. Rev Med Chile 2005; 33: 1465-71.

4.- Florin T A, Zaoutis T E, Zaoutis L B. Beyond cat scratch disease: widening spectrum of Bartonella henselae infection. Pediatrics 2008; 121: e1413-25.

5.- Vásquez $P$, Chanqueo L, García P, Poggi H, Ferrés M, Bustos M, et al. Angiomatosis bacilar por Bartonella quintana en un paciente con infección por virus de inmunodeficiencia humana. Rev Chilena Infectol 2007; 24: 155-9.

6.- Miranda R, Stambuk J, Vial M T, Miranda A. Peliosis hepática focal: caso clínico radiológico. Rev Med Chile 2010; 138: 1135-9.

7.- Dehio C. Bartonella-host-cell interactions and vascular tumour formation. Nat Rev Microbiol 2005; 3: 621-31.

8.- Margileth A M. Recent advances in diagnosis and treatment of cat scratch disease. Curr Infect Dis Rep 2000; 2: 141-6.

9.- Nowotny N, Deutz A, Fuchs K, Schuller W, Hinterdorfer F, Auer H, et al. Prevalence of swine influenza and other viral, bacterial, parasitic zoonoses in veterinarians. J Infect Dis 1997; 176: 1414-5.

10.- Kumakasa K, Arashima Y, Yanai M, Hosokawa N, Kawano K. Survey of veterinary professionals for antibodies to Bartonella henselae in Japan. RinsHo Byori 2001; 49: 906-10.

11.- Rath P M, von Recklinghausen G, Ansorg R. Seroprevalence of immunoglobulin G antibodies to Bartonella henselae in cat owners. Eur J Clin Microbiol Infect Dis 1997; 16: 326-7.

12.- Abarca K, Rivera M, Vial P, Ferrés M, García C, Oddó D. Enfermedad por arañazo de gato, informe de ocho casos. Honduras Pediátrica 1998; 19: 13-6.

13.- Eymin G, Zapata A, Andrade M, Aizman A, Rojas L, Ragabliati R. Enfermedad por arañazo de gato. Características clínicas y de laboratorio en pacientes adultos hospitalizados por fiebre o adenopatías. Rev Med Chile
2006; 134: 1243-8.

14.- Wolff E, Muñoz M, Zapata C, Ledermann W. Enfermedad por arañazo de gato complicada con compromiso sistémico, osteomielitis osteovertebral y absceso paravertebral. Rev Chilena Infectol 2000; 17: 332-9.

15.- Täger M, Zamorano R J. Osteomielitis, una manifestación inusual de la enfermedad por arañazo de gato. Rev Chilena Infectol 2000; 17: 326-31.

16.- Ladrón de Guevara D, Lobo G, Miranda M, Wu E, Muñoz M A, Pérez A, et al. Forma atípica de enfermedad por arañazo de gato: Compromiso óseo en dos pacientes pediátricos detectado mediante cintigrafía ósea. Revisión de la literatura. Rev Chilena Infectol 2003; 20: 202-9.

17.- Navarrete M, Tager M, Wenzel M S, Podestá L, Pincheira M, Zaror L. Formas atípicas de enfermedad por arañazo de gato, Valdivia. Rev Chilena Infectol 2003; 20: 257-61.

18.- Ballesteros A. Detección serológica de Bartonella henselae en gatos y sus propietarios en la ciudad de Valdivia. Tesis Méd Vet Valdivia, Chile. Universidad Austral de Chile 2000; 34.

19.- Ferrés M, Abarca K, Prado P, Montecinos L, Navarrete M, Vial P. Prevalencia de anticuerpos contra Bartonella henselae en niños, en adolescentes y en una población de riesgo ocupacional en Chile. Rev Med Chile 2006; 134: 863-7.

20.- Abarca K, Winter M, Marsac D, Palma C, Contreras A, Ferrés M. Exactitud y utilidad diagnóstica de la IgM en infecciones por Bartonella henselae. Rev Chilena Infectol 2013; 30: 125-8.

21.- Noah D L, Kramer C M, Verbsky M P, Rooney J A, Smith K A, Childs J E. Survey of veterinary professionals and other veterinary conference attendees for antibodies to Bartonella henselae and B. quintana. J Am Vet Med Assoc 1997; 210: 342-4.

22.- Maruyama S, Boonmar S, Morita Y, Sakai T, Tanaka S, Yamaguchi F, et al. Seroprevalence of Bartonella henselae and Toxoplasma gondii among healthy individuals in Thailand. Thailand J Vet Med Sci 2000; 62: 635-7.

23.- Jackson L A, Perkins B A, Wenger J D. Cat scratch disease in the United States: an analysis of three national databases. Am J Public Health 1993; 83: 1707-11.

24.- Maurin M, Raoult D. Bartonella infections: diagnostic and management issues. Curr Opin Infect Dis 1998; 11: 189-93.

25.- Zangwill K M, Hamilton D H, Perkins B A, Regnery R L, Plikaytis B $\mathrm{D}$, Hadler J L, et al. Cat scratch disease in Connecticut. Epidemiology, risk factors, and evolution of a new diagnostic test. N Engl J Med 1993; 329: 8-13.

26.- Henn J, Liu C, Kasten R, Van Horn B, Beckett L, Kass P, et al. Seroprevalence of antibodies against Bartonella species and evaluation of risk factors and clinical signs associated with seropositivity in dogs. Am J Vet Res 2005; 66: 688-94.

27.- Yamanouchi H, Izumikawa K, Hisamatsu T, Yoshinaga M, Sasaki E, Izumikawa $\mathrm{K}$, et al. A case of Bartonella henselae infection from a dog. Kansenshogaku Zasshi 2004; 78: 270-3.

28.- Ayllón T, Diniz P P, Breitschwerdt E B,Villaescusa A, Rodríguez-Franco F, Sainz A. Vector-borne diseases in client-owned and stray cats from Madrid, Spain. Vector Borne Zoonotic Dis 2012; 12: 143-50.

29.- Cornejo W, Vizcarra H. Bartonella henselae: nuevo patógeno en humanos. Lima, Perú. Anales de la Facultad de Medicina, Universidad Nacional Mayor de San Marcos 1999; 60: 281-92. 